\title{
Optimizing the Mean and Variance of Bead Geometry in the Wire + Arc Additive Manufacturing Using a Desirability Function Method
}

Jin-Soo Cho

Hanyang University - Seoul Campus

Dong-Hee Lee ( $\nabla$ dhee@skku.edu )

Sungkyunkwan University College of Engineering https://orcid.org/0000-0001-8549-8992

Gi-Jeong Seo

Tennessee Tech University

Duck-Bong Kim

Tennessee Tech University

Seung-Jun Shin

Hanyang University - Seoul Campus

\section{Research Article}

Keywords: Wire + arc additive manufacturing process, TZM, Bead Geometry, Desirability Function Method, Response Surface Methodology

Posted Date: November 18th, 2021

DOI: https://doi.org/10.21203/rs.3.rs-1041441/v1

License: (c) (i) This work is licensed under a Creative Commons Attribution 4.0 International License.

Read Full License 


\title{
Optimizing the mean and variance of bead geometry in the wire + arc additive manufacturing using a desirability function method
}

\author{
Jin-Soo Cho ${ }^{\mathrm{a}}$, Dong-Hee Lee ${ }^{\mathrm{b} *}$, Gi-Jeong Seo ${ }^{\mathrm{c}}$, Duck-Bong Kim ${ }^{\mathrm{c}}$, Seung-Jun Shin ${ }^{\mathrm{a}}$ \\ ${ }^{a}$ Division of Interdisciplinary Industrial Studies, Hanyang University, 222 Wangsimni-ro, \\ Seongdong-gu, Seoul, 04763, Republic of Korea \\ ${ }^{b}$ Department of Industrial Engineering, Sungkyunkwan University, 2066 Seobu-ro, Jangan-gu, \\ Suwon, 16419, Republic of Korea
}

${ }^{c}$ Department of Manufacturing and Engineering Technology, Tennessee Technological University, 1 William L Jones Drive, Cookeville, TN 38505, United States

*Corresponding author. Telephone \& Fax: +82-31-290-7609; e-mail: dhee@skku.edu; ORCID: https://orcid.org/0000-0001-8549-8992 


\begin{abstract}
Wire + arc additive manufacturing (WAAM) is an arc welding process that uses nonconsumable tungsten electrodes to produce the weld. The material used in this study is a titanium, carbon, zirconium, and molybdenum (TZM) alloy that is physically and chemically stable and has good performance for use as a welding and high-temperature heating element. However, the price is higher than that of other materials. Because welding cannot be modified after manufacturing, economic losses are high in the case of a defective product. Therefore, it is important to find the best welding settings for the target bead geometry during welding. In this study, welding experiments are designed based on a central composite design, and single-layer WAAM is performed using a TZM material. Consequently, we obtain 17 beads and measure the height, width, as well as left and right toe angles, which represent the geometry of the beads. Based on the measured geometry, we obtain the optimal settings for the WAAM parameters whereat the mean of each geometry is close to its target value and its variance is minimized by using a desirability function method. Furthermore, we conduct additional experiments to validate the optimal settings that we obtain. We compare the predicted and actual geometry values and find that they are quite close. This result indicates that valid optimal settings for the process parameters can be obtained via the proposed method.
\end{abstract}

\title{
Highlights
}

- Massive measurement data for the TZM bead geometry were collected using a coordinate measuring machine

- The mean and variance of the TZM bead geometry were modeled as second-order models using response surface methodologies

- The optimal settings for the Wire + arc additive manufacturing process parameters were obtained via a desirability function method

\section{Keywords}

Wire + arc additive manufacturing process, TZM, Bead Geometry, Desirability Function Method, Response Surface Methodology 


\section{Introduction}

Welding is a typical manufacturing process whereby metals are joined by using high heat to melt the metals together and allowed to cool thereafter. Wire + arc additive manufacturing (WAAM) is a typical welding process that uses non-consumable tungsten electrodes. Notably, WAAM is used in various industries, including aerospace, construction, auto repair, art, nuclear, auto renovation, and shipping, because of its ability to produce large-sized metallic parts with a high deposition rate, low equipment cost, and high material utilization.

In this study, we apply WAAM to TZM, which is an alloy of titanium $(0.5 \%)$, carbon $(0.02 \%)$, zirconium (0.08\%), molybdenum (99.40\%). The raw TZM alloy is transformed into a weld bead through the WAAM process. Figure 1 shows examples of TZM beads. Moreover, TZM has a physically stable performance with 1,400 degrees of recrystallization and good performance for welding and high-temperature heating, making it widely used in the real world for several applications, including nozzles, valve bodies, gas piping, gated tube molds, and hot electric heat sinks. However, TZM is an expensive metal; thus, WAAM should be carefully conducted to prevent defective TZM beads. It is also important for the bead to have good bead geometry, where the height, length, and angle to the surface are desirable parameters. Compared to the lower image, the upper image shows a desirable bead geometry, where the height, length, and angles are close to the targets and have low variability across the bead. The targets of the bead geometry are user-defined parameters, and the details of the parameters are explained in Section 3.

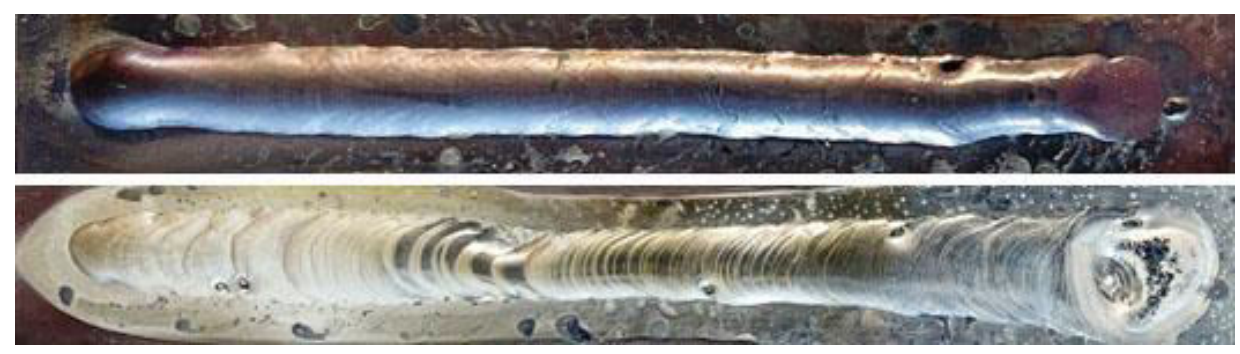

Fig. 1 Examples of TZM beads

In the WAAM process, the bead geometry is affected by certain process parameters, such as 
the current, welding speed, and feed rate. To achieve good bead geometry, these process parameters should be maintained at predetermined optimal settings during the WAAM process. Response surface methodology (RSM) can be used for this purpose. Notably, RSM is a bundle of statistical and optimization techniques that empirically studies the relationship between a response variable and the input variables that affect it. The ultimate goal of RSM is to determine the optimal settings of the input variables to optimize the response variable. In terms of RSM, the WAAM process parameters correspond to the input variables, and the bead geometry parameters correspond to the response variables. The geometry can be represented by height, length, and left and right angles, and they are considered as response variables.

Several studies have been conducted on bead geometry and welding process parameters. These studies have all aimed at determining the optimal settings for the welding process parameters. Kim et al. [1] proposed a method to optimize the welding process parameters of a gas metal arc process using genetic algorithms and RSM. In this method, the optimal settings for the wire feed rate and welding voltage were obtained to optimize the height, width, and penetration of the bead. Dey et al. [2] suggested the use of a genetic algorithm and penalty function to obtain the optimal settings for voltage, current, and welding speed to optimize the height, width, and penetration of the bead. Geng et al. [3] suggested a method for optimizing wire feeding in a WAAM process. They improved the deposition accuracy in WAAM when the wire was fed in a sidewise direction. A mathematical model was developed to calculate the wire flying distance in the arc zone, according to which the displacement compensation was designed to ensure size accuracy. Benyounis et al. [4] investigated the effect of three welding process parameters - laser power, welding speed, and focal point position — on three responses - penetration, welded zone width, and heat-affected zone width — using RSM. They built regression models to explain the effects and obtained the optimal settings of the three process parameters whereat the three responses were desirable. Gunaraj and Murugan [5] conducted a study to predict the weld bead quality in submerged arc welding and determine the essential parameters for the desired quality and process optimization using RSM.

Most of the above-mentioned methods were used in an attempt to determine the optimal settings of the process parameters to optimize the multiple response variables representing the bead geometry. They are typical examples of multiple response problems in the welding 
process field. Multiple response surface optimization (MRSO) is a research field for solving the multiple response problem based on the RSM framework [6]. In MRSO, multiple responses are often in conflict. That is, in many multi-response problems, improving one response is likely to worsen one or more other responses [7]. In such cases, it is important to consider the tradeoffs between the multiple response variables and find an optimal setting of the input variables whereat the multiple response variables are optimally compromised. To find the optimally compromised setting, it is necessary to reflect a process engineer's preference information regarding the multiple responses. Most of the existing methods have a disadvantage in that they do not systematically reflect the process engineer's preference information regarding the multiple process. In addition, they did not consider the variability of the bead geometry, which is critical to bead quality.

Thus, we suggest using a desirability function method to solve the multiple response problem of the WAAM process. The desirability function method, suggested by Derringer and Suich [8], is a representative approach for obtaining a compromised optimal setting by systematically reflecting the process engineer's preference information. We use the desirability functions to obtain the optimal settings of the WAAM process parameters (i.e., current, welding speed, and feed rate), where the four response variables (i.e., height, length, and left and right angles) are optimized simultaneously. At the optimal settings, the four response variables are close to their target values. Additionally, the variability of the four responses across the bead is stable, which means that the variances of the four response variables are low.

To obtain the optimal settings, we first conduct experiments for the WAAM process based on RSM to investigate the relationship between the input variables and the mean of each response. In addition, the relationship between the input variables and the standard deviation of each response is investigated. Based on the experimental data, we build regression models for the mean and standard deviation for each response variable. Thereafter, we construct individual desirability functions for the mean and standard deviation of the four response variables and aggregate the individual desirability functions into an overall desirability function. Finally, the optimal settings of the input variables are obtained by maximizing the overall desirability function. 
As previously mentioned, the proposed method is advantageous in that it systematically reflects the process engineer's preference information to obtain the optimal settings where the four response variables are compromised. In addition, the proposed method has a significant industrial contribution, as it is the first approach toward optimizing the TZM bead geometry in the WAAM process. The TZM bead geometry is difficult to measure in practice because the bead should be cut to measure, and it is an expensive metal such that cutting requires high cost and effort. We conduct experiments and measure the TZM bead geometry using a coordinate measuring machine (CMM). Based on the measured data, the proposed method establishes the relationship between the bead geometry and the WAAM process parameters. Ultimately, the optimal settings of the WAAM process parameters are obtained using the desirability function method.

The remainder of this paper is organized as follows: In Section 2, we review RSM and the desirability function method. In Section 3, the WAAM process optimization using the desirability function method is explained. We describe the results and validation of our case study in Section 4, while the concluding remarks are provided in Section 5.

\section{Literature review}

Response surface methodology, first proposed by Box and Wilson [9], is a quality engineering approach utilized for process optimization, and it is a statistical and optimization tool to systematically derive optimal process settings by analyzing the relationship between input and response variables. The ultimate purpose of RSM is to determine the optimal settings for the input variables that optimize the response. Usually, RSM consists of three stages: data collection, modeling, and optimization $[9,10]$.

Typically, RSM is used for modeling problems in which the responses of interest are affected by a set of variables. However, in reality, there are more problems with multiple responses than with single ones. Therefore, considering multiple response variables is common in product or process development. A common approach to multi-response optimization is to construct empirical models for multiple responses under the RSM framework and analyze the empirical models to obtain the optimal settings for the input variables. This approach is 
referred to as MRSO [6]. The multi-response problem can be formally expressed as follows:

$$
\begin{gathered}
\text { Optimize }\left[\hat{y}_{1}(\mathbf{x}), \hat{y}_{2}(\mathbf{x}), \ldots, \hat{y}_{m}(\mathbf{x})\right] \\
\text { subject to } \mathbf{x} \in \mathbf{\Omega}
\end{gathered}
$$

where $\mathbf{x}$ is a vector of input variables, $\boldsymbol{\Omega}$ is a feasible region of $\mathbf{x}, \hat{y}_{j}(j=1,2, \ldots, m)$ is the fitted response function of the $j^{\text {th }}$ response variable $y_{j}$, and $m$ is the number of responses.

When fitting the response surface functions, central composite design (CCD) is the most widely used approach. The CCD experimental points consist of the center, factorial, and axial points. An important CCD property is that the expected values of the variance for the response values at points equal to the center point are equal. Therefore, CCD is an efficient way to obtain the maximum effect with fewer experiments. Several studies have been conducted to optimize weld bead geometry using CCD. Recently, Saha and Waghmare [11] conducted a study to optimize the laser power, laser scan speed, and local point position in laser beam welding using sub-millimeter-thick SS316 sheets. This study designed and performed a total of 20 experimental points of 8 factorial, 6 axial, and 6 center points using CCD. Panaskar and Terkar [12] used CCD to optimize the friction stir welding process parameters for AA6063-ETP copper welding. As input variables in the CCD process, they used the tool rotational speed, tool traverse speed, and thickness of the inter-pillar zinc foil, and the experiment was performed by constructing 60 experimental points according to the CCD process.

An important problem in multi-response optimization is resolving the conflicts between multiple responses. Typical optimization methods include the desirability function method [10] and the loss function method [13,14]. The desirability function method is one of the approaches to optimization, as first introduced by Derringer and Suich [8]. The desirability function method is widely used to optimize multiple response problems. The methodological basis for the desirability function approach is to transform the estimated response on a quality characteristic to a value, known as desirability, for each response. The geometric mean of all the individual desirability values is then used to represent the overall desirability [15]. 
The establishment of a desirability function depends on the type of each response variable. There are three types: nominal-the-best (NTB), larger-the-better (LTB), and smaller-the-better (STB). Notably, NTB has high satisfaction when the response value is close to a specific target value. The higher the response value, the higher the satisfaction level of LTB. In contrast, STB shows higher satisfaction with smaller response values. The construction of the desirability function is constructed according to Equations (1), (2), and (3) for NTB, LTB, and STB, respectively. Equations (1), (2), and (3) include several parameters that represent the engineer's preference for multiple responses: $T_{j}$ is the target value of $y_{j} ; y_{j}^{\text {min }}$ and $y_{j}^{\max }$ are the lower and upper limits of $y_{j}$, respectively; and $s_{j}$ and $t_{j}$ determine the shapes of $d_{j}$. When $s_{j}\left(t_{j}\right)$ is $1, d_{j}$ is expressed linearly. If $s_{j}\left(t_{j}\right)$ has a value between 0 and 1 , the desirability function is expressed as a nonlinear function in a concave form. If $s_{j}\left(t_{j}\right)$ has a value greater than 1 , it is expressed as a convex function. Process engineers can represent their preferences for multiple responses through these parameters. Thus, these parameters are called preference parameters.

$$
\begin{aligned}
& d_{j}(\mathbf{x})=\left\{\begin{array}{lc}
0, & \text { if } \hat{y}_{j}(\mathbf{x}) \leq y_{j}^{\min } \text { or } \hat{y}_{j}(\mathbf{x}) \geq y_{j}^{\max } \\
\left(\frac{\hat{y}_{j}(\mathbf{x})-y_{j}^{\min }}{T_{j}-y_{j}^{\min }}\right)^{s_{j}}, & \text { if } y_{j}^{\min }<\hat{y}_{j}(\mathbf{x})<T_{j} \\
\left(\frac{y_{j}^{\max }-\hat{y}_{j}(\mathbf{x})}{y_{j}^{\max }-T_{j}}\right)^{t_{j}}, & \text { if } T_{j} \leq \hat{y}_{j}(\mathbf{x})<y_{j}^{\max }
\end{array}\right. \\
& d_{j}(\mathbf{x})=\left\{\begin{array}{c}
0, \\
\left(\frac{\hat{y}_{j}(\mathbf{x})-y_{j}^{\min }}{y_{j}^{\max }-y_{j}^{\min }}\right)^{s_{j}}, \\
1,
\end{array}\right. \\
& \text { if } \hat{y}_{j}(\mathbf{x}) \leq y_{j}^{\min } \\
& \text { if } y_{j}^{\min }<\hat{y}_{j}(\mathbf{x})<y_{j}^{\max } \\
& \text { if } \hat{y}_{j}(\mathbf{x}) \geq y_{j}^{\max } \\
& d_{j}(\mathbf{x})=\left\{\begin{array}{c}
1, \\
\left(\frac{y_{j}^{\max }-\hat{y}_{j}(\mathbf{x}),}{y_{j}^{\max }-y_{j}^{\min }}\right)^{t_{j}}, \\
0,
\end{array}\right.
\end{aligned}
$$

When all the individual desirability functions are constructed for each response, they are aggregated into an overall desirability function, and the geometric mean or weighted geometric mean is often used for the aggregation, as shown in Equation (4). 


$$
D(\mathbf{x})=\left(\prod_{j=1}^{m} d_{j}(\mathbf{x})^{w_{j}}\right)^{\frac{1}{\sum_{j=1}^{m} w_{j}}}
$$

where $w_{j}$ is the relative weight of $y_{j}$. Relative weights can be given differently depending on the user's preference [6]. Finally, the optimal setting of the input variables is obtained by maximizing the overall desirability function.

\section{Optimization of the WAAM process}

In this study, the optimal setting of the WAAM process parameters is determined using the desirability function method, which considers not only the mean but also the standard deviation of the bead geometry.

\subsection{Overview of the WAAM process}

In this section, the input and response variables of the WAAM process for welding the TZM alloy are introduced. Thereafter, the overall procedure for optimizing the WAAM process is presented. We consider three parameters for the input variables: current, welding speed, and feed rate. Figure 2 shows a schematic diagram of the three input variables in the WAAM process. The current in welding (ampere) is the welding parameter representing the flow of electrons that crosses the arc gap between the electrode end and the metal being welded. The greater the electrical resistance, the greater the heat generated by the arc, and the higher the temperature. The current affects weld penetration. As the weld current increases, both the deposition speed and penetration depth increase. Excessive current can cause electrode wires to overheat, thereby causing arc instability, poor welding quality, disconnection, or bowling. The current value must be controlled to an appropriate level to maintain the stability of the arc. 


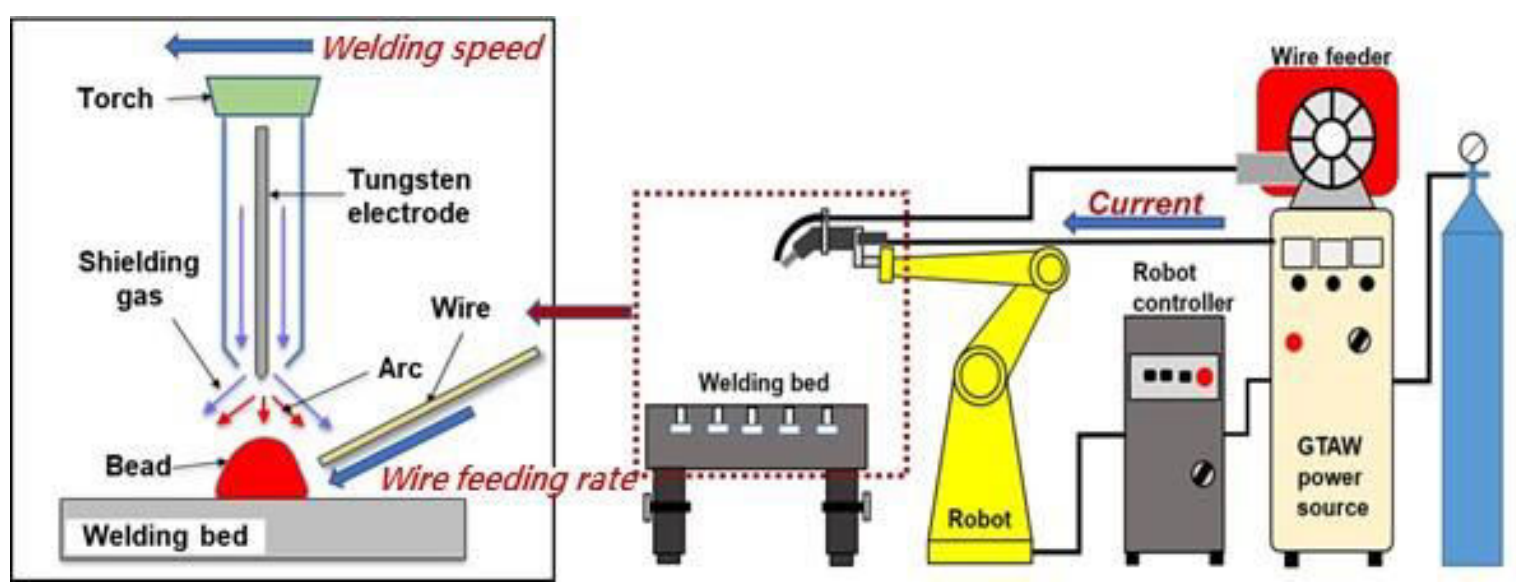

Fig. 2 Schematic diagram of the three input variables in the WAAM process

The welding speed is that at which the electrode moves relative to the junction. In this study, it is expressed in centimeters per minute $(\mathrm{cm} / \mathrm{min})$. Usually, thick materials use slow speed, and thin materials use fast speed. The welding speed adversely affects the weld bead size. A fast (slow) welding speed reduces (increases) the weld size. A very low welding speed can cause the arc to collide with a thick layer of molten metal, thereby reducing penetration.

The feed rate is the speed at which the material in the wire is transferred to the weld. It is also expressed in $\mathrm{cm} / \mathrm{min}$. The feed rate is directly related to the weld current and current growth as the wire feed speed increases. The faster the wire is supplied, the better the contacts are delivered, and more amps pass through the wire to increase the heat strength. If the wire speed is too high, the arc may start incorrectly, and a wide bead shape may appear.

We consider four response variables representing the bead geometry: height, width, left toe angle, and right toe angle. The details of the four response variables are explained in Section 3.2. Figure 3 shows the overall flow of the study. It consists of seven steps, and they can be mapped into the three RSM stages (i.e., data collection, model building, and optimization). Steps 1 and 2 can be mapped to data collection. Step 3 involves model building. Steps 4-7 are used for optimization. The details of each step are explained in Section 3.2. 


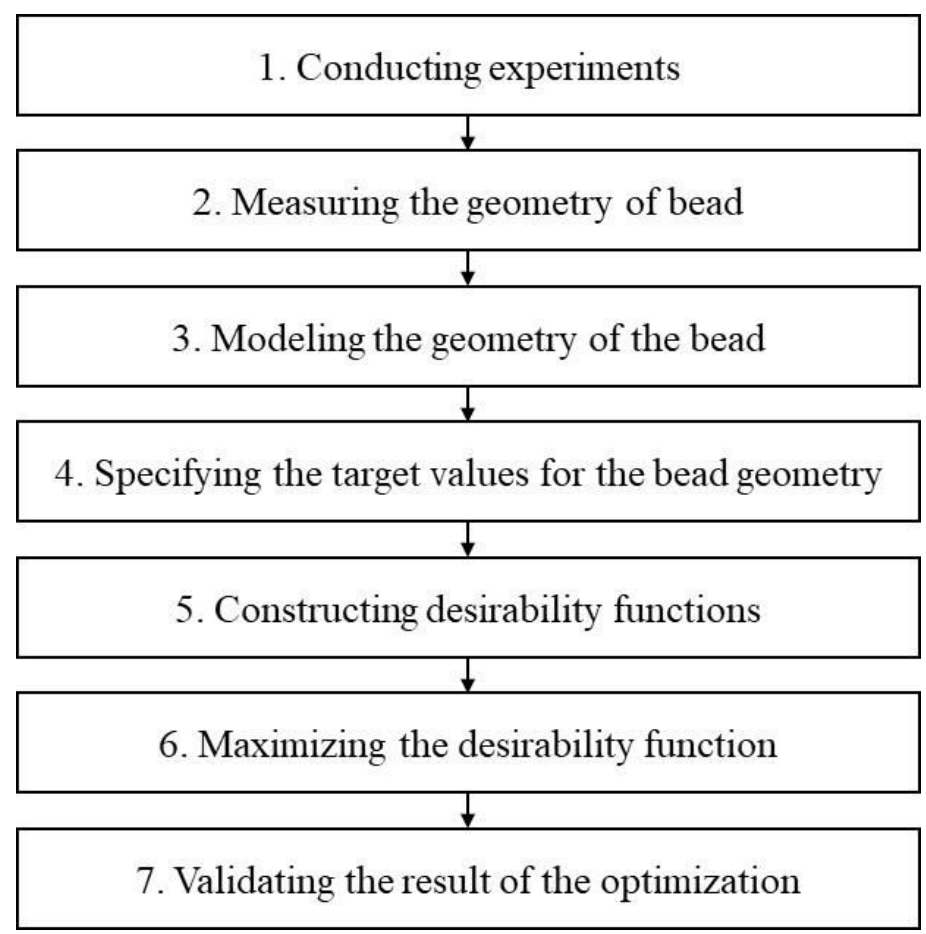

Fig. 3 Overall flow of this study

\subsection{Step-by-step procedure of WAAM optimization}

\subsubsection{Step 1: Conducting experiments}

Welding experiments are conducted using WAAM to generate TZM beads, as described in Figure 4. The experiments are conducted as "bead on plate tests," which generate TZM beads on a plate according to experimental conditions.

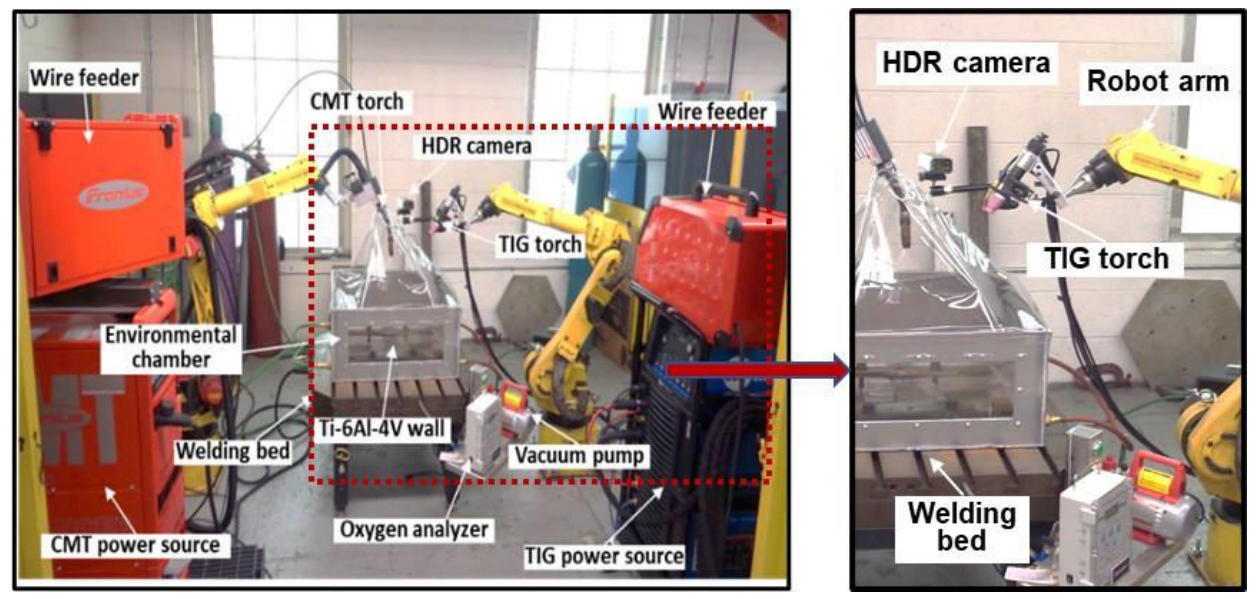

Fig. 4 Experimental facility

In addition to the three input variables, the other process parameters are determined as 
conventional values. The gas used for welding was a mixture of $70 \%$ argon and $30 \%$ helium. The substrate used as a test plate for welding was based on a base metal and was $100 \mathrm{~mm} \times$ $100 \mathrm{~mm}$ in size. A 5-mm-diameter wire was used. To minimize external factors in the experiment and ensure uniform settings, the temperature of the substrate was maintained between $50-55^{\circ} \mathrm{C}$ during each experiment.

The experimental factors include the process parameters, current, welding speed, and feed rate of welding. According to $\mathrm{CCD}$, three levels were set $[-1,0,1]$, and the actual experimental values were set using empirical data from experts in the welding field. Table 1 presents the actual experimental values of the three factors for the three coded values $[-1,0$, 1]. According to $\mathrm{CCD}$, we set eight experimental points as factorial points, six as axial points, and three as center points. Thus, a total of 17 experiments were conducted according to the design points listed in Table 2.

Table 1 Factors and levels for the center composite design

\begin{tabular}{cccc}
\hline Level & Current & Welding Speed & Feed Rate \\
\hline-1 & 275 & 10 & 100 \\
0 & 300 & 15 & 150 \\
+1 & 325 & 20 & 200 \\
\hline
\end{tabular}

Table 2 Central composite design of TZM welding

\begin{tabular}{c|ccc|ccc}
\hline \multirow{2}{*}{$\begin{array}{c}\text { Experiment } \\
\text { Number }\end{array}$} & \multicolumn{3}{|c|}{ Coded value } & \multicolumn{3}{c}{ Real value } \\
\cline { 2 - 7 } & Current & $\begin{array}{c}\text { Welding } \\
\text { Speed }\end{array}$ & Feed Rate & Current & $\begin{array}{c}\text { Welding } \\
\text { Speed }\end{array}$ & Feed Rate \\
\hline 1 & -1 & -1 & -1 & 275 & 10 & 100 \\
2 & 1 & -1 & -1 & 325 & 10 & 100 \\
3 & -1 & 1 & -1 & 275 & 20 & 100 \\
4 & 1 & 1 & -1 & 325 & 20 & 100 \\
5 & -1 & -1 & 1 & 275 & 10 & 200 \\
6 & 1 & -1 & 1 & 325 & 10 & 200 \\
7 & -1 & 1 & 1 & 275 & 20 & 200 \\
8 & 1 & 1 & 1 & 325 & 20 & 200 \\
9 & -1.6818 & 0 & 0 & 258 & 15 & 150 \\
10 & 1.6818 & 0 & 0 & 342 & 15 & 150 \\
11 & 0 & -1.6818 & 0 & 300 & 7 & 150 \\
12 & 0 & 1.6818 & 0 & 300 & 23 & 150 \\
13 & 0 & 0 & -1.6818 & 300 & 15 & 66
\end{tabular}




\begin{tabular}{c|ccc|ccc}
14 & 0 & 0 & 1.6818 & 300 & 15 & 234 \\
\hline 15 & 0 & 0 & 0 & 300 & 15 & 150 \\
16 & 0 & 0 & 0 & 300 & 15 & 150 \\
17 & 0 & 0 & 0 & 300 & 15 & 150 \\
\hline
\end{tabular}

The experiment was conducted using single-layer welding. An image of the upper surface of the bead with the completed welding experiments is presented in Table 3. It can be confirmed that the bead geometry varies depending on the process parameters of the welding experiments.

Table 3 Bead surface images

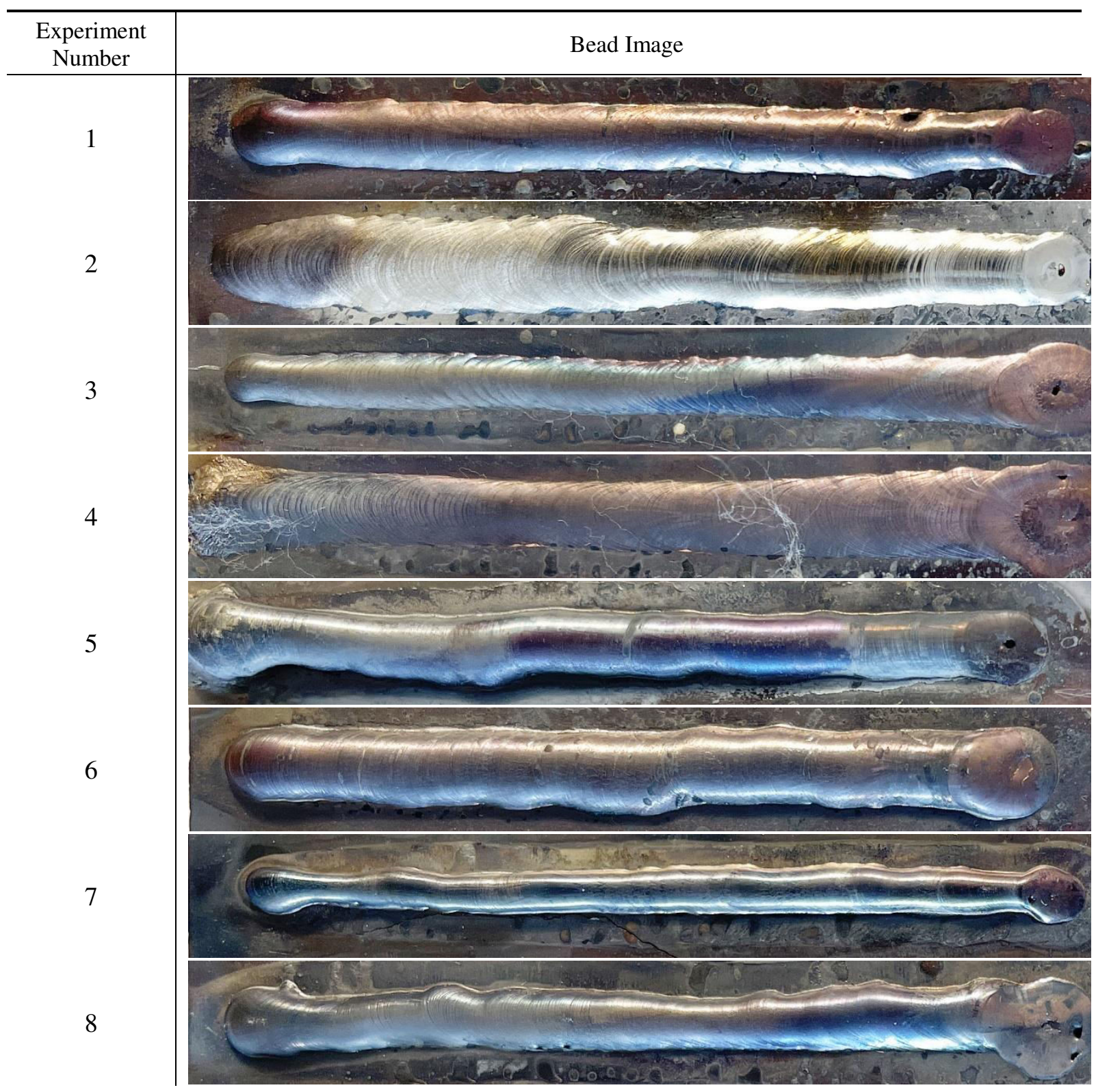



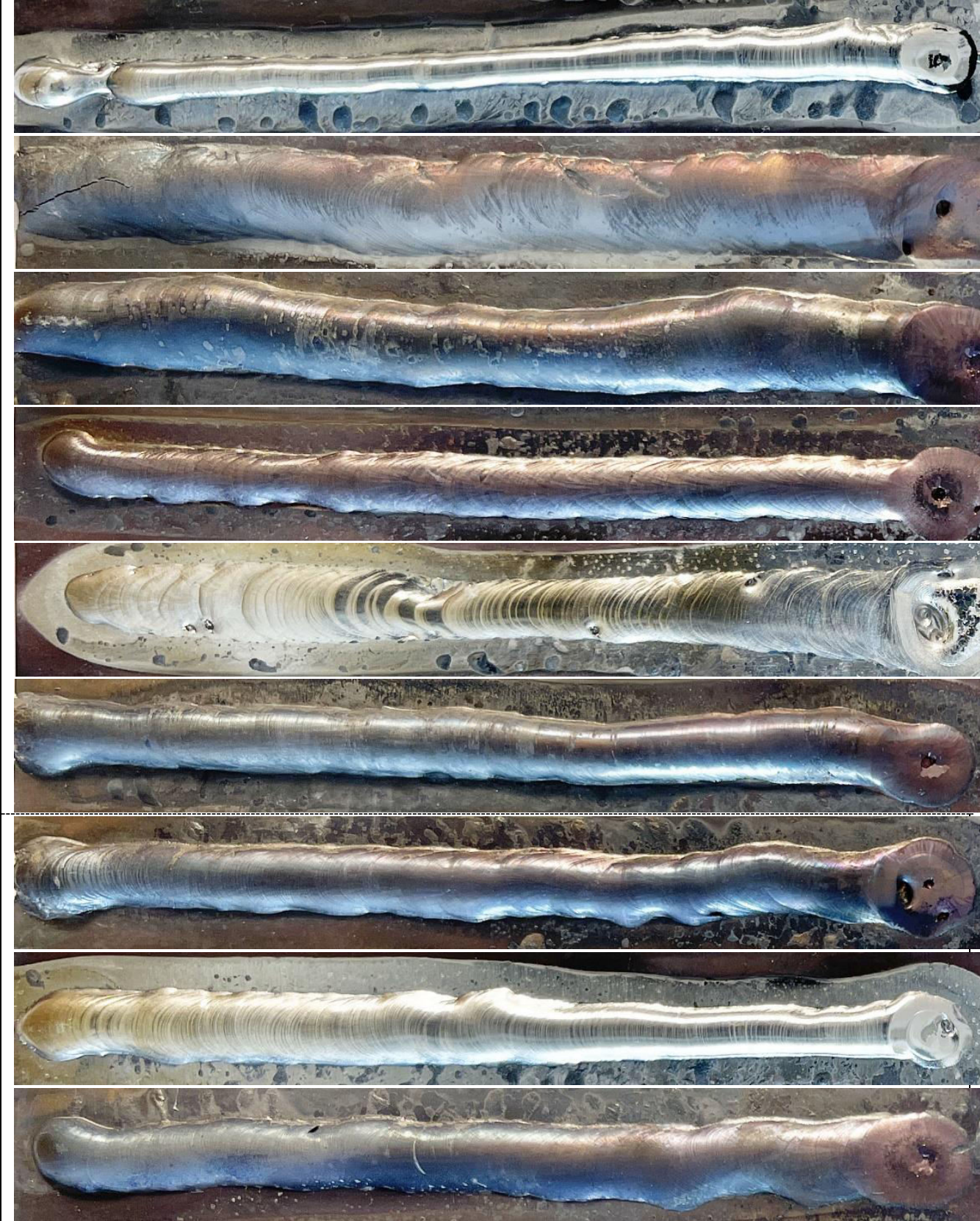

\subsubsection{Step 2: Measuring the geometry of the bead}

As previously mentioned, we consider four response variables that represent the geometry of the TZM beads. In Step 1, we obtained 17 TZM beads. In this step, we measure the four response variables at 100 evenly divided points on each bead. We have 17 beads, 100 points on each bead, and 4 response variables; thus, a total of 6,800 (i.e., $17 \times 100 \times 4$ ) values for the response variables should be measured. It is almost impossible to manually measure 6,800 values; thus, we develop an automatic measurement program using the MATLAB 
2019a software. The details of the program development are presented as follows:

First, we exclude the start and end parts of the beads. When TZM bead welding is performed, there is an instability that does not reliably reflect the welding settings selected by the user at the beginning and end of the welding. This is due to external factors, such as arc, temperature, and wire supply changes. The start and end areas of the beads are sensitive to these external factors. Thus, we exclude these areas during geometry measurement. The excluded area is approximately less than $10 \%$ of the total bead

Second, approximately $90 \%$ of the bead is divided into 100 points, and the 4 response values are measured at each point. For this purpose, we use a CMM to obtain a cross-sectional image at each point and convert this image into coordinate information. The CMM scans the beads to obtain a cross-sectional image and converts it into coordinate information. When scanning beads using CMM, a matte spray is sprayed on top of a single-sided bead to prevent measurement errors and noise, followed by matte treatment. After scanning, the scanned cross-sectional image is converted into XYZ coordinate information. The $\mathrm{x}$ coordinate indicates the location where the cross-sectional image is scanned at the bead. Once $\mathrm{x}$ is given, the $\mathrm{y}$ and $\mathrm{z}$ coordinates are generated from the scanned cross-sectional image at $\mathrm{x}$. Figure 5 shows an example of the XYZ coordinate information obtained from the cross-sectional image.

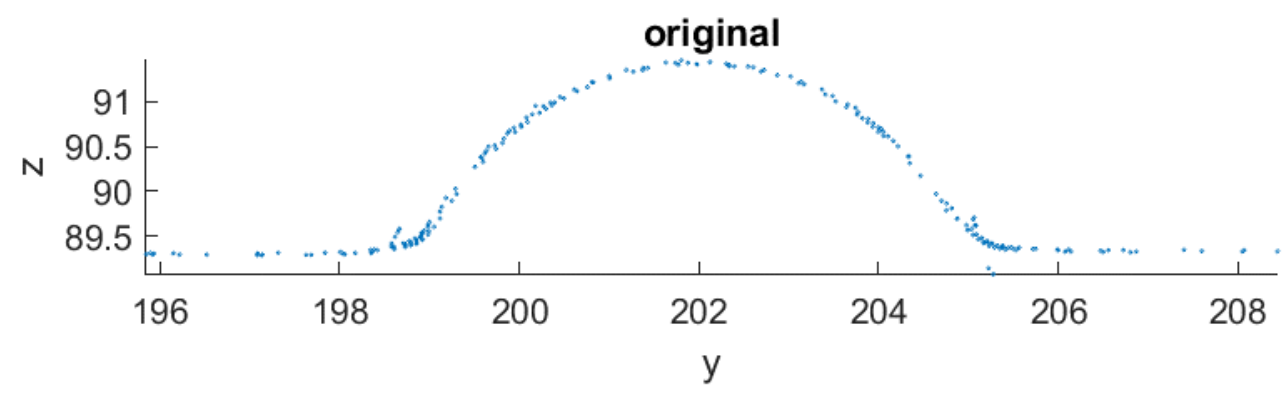

Fig. 5 Example of the coordinates of the cross-sectional image extracted from bead No. 8

Third, based on the coordinate information, interpolation is conducted on the coordinate information. One of the measurement-related difficulties is that coordinate points are not densely present, as shown in Figure 5. Each point is fitted with tertiary spline interpolation to form a curve. Tertiary spline interpolation is a method of obtaining an n-order polynomial 
past that n-point, given a discontinuous n-point. In other words, the sub-intercept between each point is connected in a tertiary polynomial, and all the segments are combined to make differentiation possible. The third spline interpolation method, which exists as a built-in function in the MATLAB 2019a software, is applied. The left-hand figure in Figure 6 is the image during the third spline interpolation process, and the right-hand figure is the image connected in a curved form after applying the interpolation method.
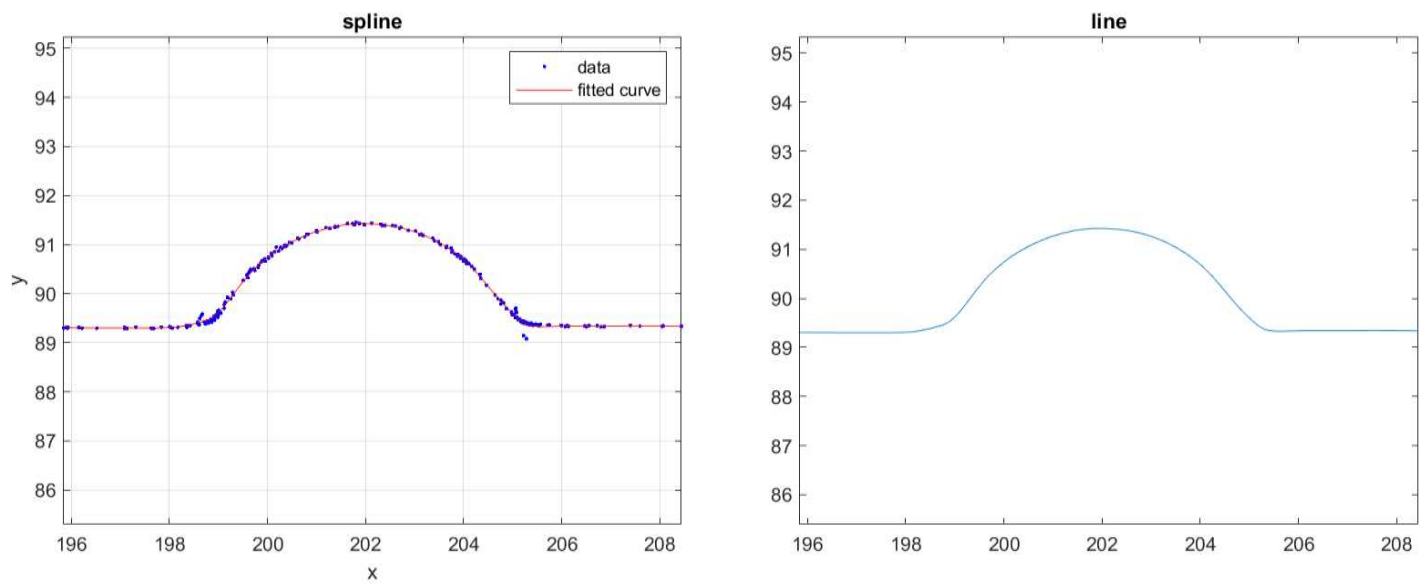

Fig. 6 Example of the spline interpolation process

Fourth, after applying the spline interpolation method, we measure the height, width, right toe angle, and left toe angle of the bead shown in the interpolated images. It is easy to measure the height and width of the beads from the interpolated images. However, toe angles cannot be measured directly because they show curvature. To measure the toe angles, we first find the point in the curve data that maximizes the curvature value. Based on this point, the toe angle is measured using a tangent line at the part corresponding to the center of the horizontal line, curve, and bead height. As shown in Figure 7, the curvature is the maximum part of the area that appears red. Based on this point, we measure the left and right toe angles using tangents. 

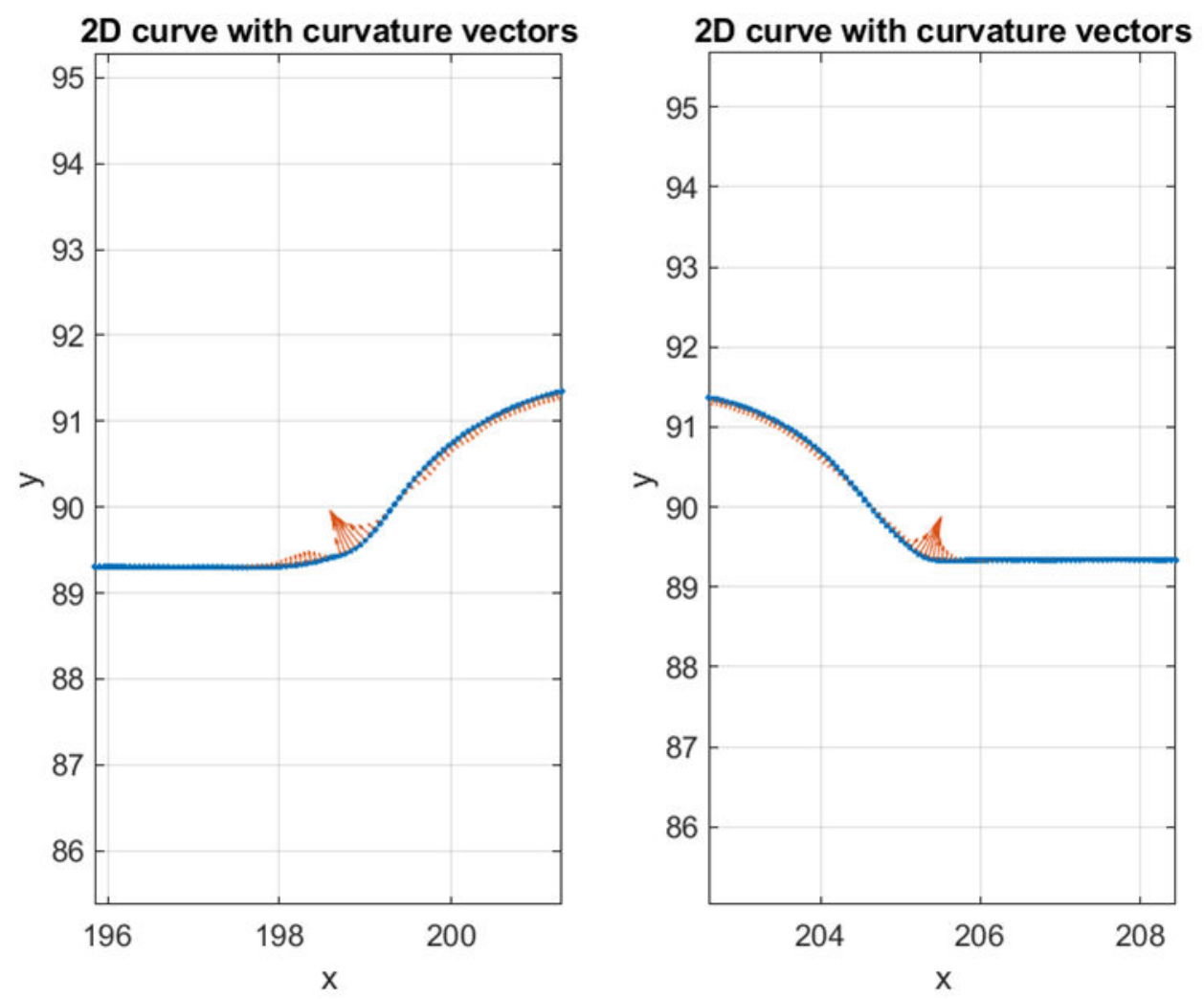

Fig. 7 Image of the process of obtaining the toe angles using curvature vectors;

left: left-hand side of the bead and right: right-hand side of the bead

Overall, the height, width, left toe angle, and right toe angle are measured at 100 points on each bead using the program we develop.

\subsubsection{Step 3: Modeling the geometry of the bead}

In Step 2, we obtain 100 values for each response variable and bead. Based on these data, we build regression models for the four response variables. The average and standard deviation values of each response at each bead are calculated, and we build regression models for the mean and standard deviation for each response variable. Therefore, we build a total of eight regression models as shown in the following expressions: $\hat{y}_{\mu 1}, \hat{y}_{\mu 2}, \hat{y}_{\mu 3}$, and $\hat{y}_{\mu 4}$ represent the fitted models for the mean of height, width, right toe angle, and left toe angle, respectively, while $\hat{y}_{\sigma 1}, \hat{y}_{\sigma 2}, \hat{y}_{\sigma 3}$, and $\hat{y}_{\sigma 4}$ represent the fitted models for the standard deviations of the height, width, right toe angle, and left toe angle, respectively, and $x_{1}, x_{2}$, and $x_{3}$ represent the coded current, welding speed, and feed rate, respectively. 


$$
\begin{aligned}
& \hat{y}_{\mu 1}=1.460-0.1315 x_{1}-0.1020 x_{2}+0.4428 x_{3}-0.0885 x_{1}{ }^{2}-0.1177 x_{2}{ }^{2}-0.0441 x_{3}{ }^{2}+0.0886 x_{1} x_{2} \\
&+ 0.0139 x_{1} x_{3}-0.0053 x_{2} x_{3} \\
& \hat{y}_{\sigma 1}=0.1484- 0.0250 x_{1}-0.0046 x_{2}+0.0201 x_{3}+0.0024 x_{1}{ }^{2}-0.0169 x_{2}{ }^{2}-0.0240 x_{3}{ }^{2}+0.0030 x_{1} x_{2} \\
&-0.0153 x_{1} x_{3}+0.0124 x_{2} x_{3} \\
& \hat{y}_{\mu 2}=6.399+-709 x_{1}-0.277 x_{2}+0.247 x_{3}-0.448 x_{1}{ }^{2}-0.335 x_{2}{ }^{2}-0.269 x_{3}{ }^{2}+0.024 x_{1} x_{2}+0.127 x_{1} x_{3} \\
&+0.006 x_{2} x_{3} \\
& \hat{y}_{\sigma 2}=0.4170+ 0.0714 x_{1}+0.0112 x_{2}-0.1617 x_{3}-0.0281 x_{1}{ }^{2}+0.0187 x_{2}{ }^{2}+0.0812 x_{3}{ }^{2}-0.0181 x_{1} x_{2} \\
&-0.0749 x_{1} x_{3}-0.0285 x_{2} x_{3} \\
& \hat{y}_{\mu 3}=152.99+ 6.614 x_{1}+4.503 x_{2}-9.177 x_{3}-3.551 x_{1}{ }^{2}-2.55 x_{2}{ }^{2}-0.864 x_{3}{ }^{2}-0.80 x_{1} x_{2}+1.52 x_{1} x_{3} \\
&-0.44 x_{2} x_{3} \\
& \hat{y}_{\sigma 3}=3.39-0.718 x_{1}-0.403 x_{2}+0.084 x_{3}+0.371 x_{1}{ }^{2}+0.374 x_{2}{ }^{2}-0.082 x_{3}{ }^{2}-0.089 x_{1} x_{2}-0.725 x_{1} x_{3} \\
&+0.281 x_{2} x_{3} \\
& \hat{y}_{\mu 4}=151.89+ 7.18 x_{1}+4.86 x_{2}-8.57 x_{3}-3.24 x_{1}{ }^{2}-2.14 x_{2}{ }^{2}-0.44 x_{3}{ }^{2}-0.39 x_{1} x_{2}+1.99 x_{1} x_{3} \\
&-0.98 x_{2} x_{3} \\
& \hat{y}_{\sigma 4}=3.210-0.123 x_{1}-0.584 x_{2}-0.045 x_{3}-0.094 x_{1}{ }^{2}+0.556 x_{2}{ }^{2}-0.303 x_{3}{ }^{2}-0.277 x_{1} x_{2}-0.506 x_{1} x_{3}+0.180 x_{2} x_{3}
\end{aligned}
$$

\subsubsection{Step 4: Specifying the target values for the bead geometry}

For optimization, the target values for the bead geometry should be specified in advance. The target values are specified by process engineers considering the purpose of the WAAM process. For example, flat and wide beads can be preferred over high and narrow beads, or vice versa. In this regard, we assume three types of ideal beads: Beads 1, 2, and 3, as shown in Figure 8. We assume that Bead 1 is the flattest and widest bead, while Bead 3 is the narrowest one. Thereafter, we set the target geometry values for the three ideal beads by interviewing field experts in charge of the WAAM process. The target geometry values of the three ideal beads are listed in Table 4.
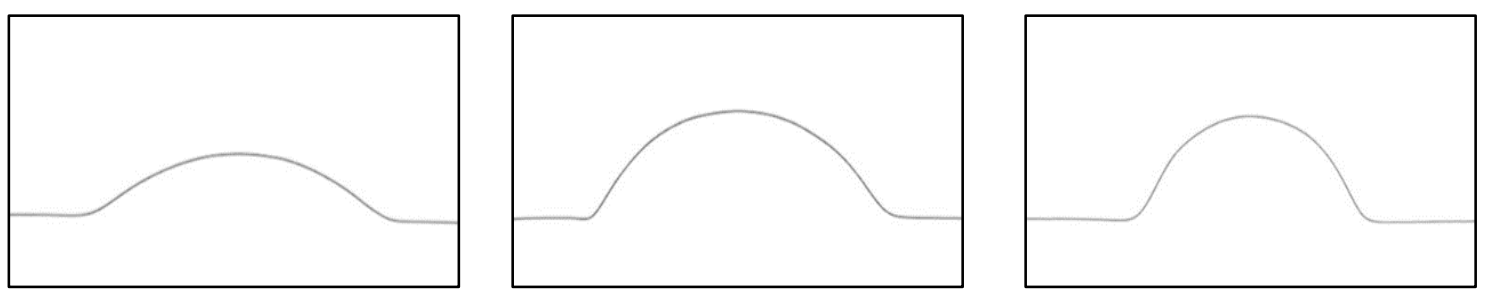

Fig. 8 Images of Beads 1,2, and 3 
Table 4 Target geometry values for Beads 1, 2, and 3

\begin{tabular}{ccccc}
\hline Geometry & Unit & Bead 1 & Bead 2 & Bead 3 \\
\hline Height & $\mathrm{mm}$ & 1.1 & 2.0 & 1.2 \\
Width & $\mathrm{mm}$ & 6.6 & 7.3 & 4.5 \\
Left toe angle & degree & 159.4 & 148.2 & 132.7 \\
Right toe angle & degree & 158.9 & 148.6 & 135.7 \\
\hline
\end{tabular}

\subsubsection{Step 5: Constructing the desirability functions}

In this study, we construct desirability functions for the mean and standard deviation of each response variable. To construct the desirability functions, $y^{\min }, T$, and $y^{\max }$ should be predetermined, as shown in Equations 1,2 , and 3. Table 5 reports the $y^{\min }, T$, and $y^{\max }$ values for the eight response surface models fitted in Step 3. The four mean response surface models (i.e., $\hat{y}_{\mu 1}, \hat{y}_{\mu 2}, \hat{y}_{\mu 3}$, and $\hat{y}_{\mu 4}$ ) are NTB types; thus, the values for all the three parameters (i.e., $y^{\min }, T, y^{\max }$ ) are specified as shown in Table 5. It should be noted that the $T$ values have already been determined in Step 4; thus, the target values given in Table 5 are the same as those in Table 4. Conversely, the four standard deviation response surface models (i.e., $\hat{y}_{\sigma 1}, \hat{y}_{\sigma 2}, \hat{y}_{\sigma 3}$, and $\hat{y}_{\sigma 4}$ ) are STB types because it is desirable to have small variability for the bead geometry. Thus, the values for only the target and upper limit are specified as shown in Table 5. Similar to Step 4, we also determine these values by liaising with the field experts in charge of the WAAM process.

Table 5 Parameter values for individual desirability functions

\begin{tabular}{|c|c|c|c|c|c|c|}
\hline Response & Model & Type & Bead & $y^{\min }$ & $T$ & $y^{\max }$ \\
\hline \multirow{6}{*}{ Height } & \multirow{3}{*}{$\hat{y}_{\mu 1}$} & \multirow{3}{*}{ NTB } & 1 & 0.4 & 1.1 & 1.5 \\
\hline & & & 2 & 1.4 & 2.0 & 2.3 \\
\hline & & & 3 & 0.9 & 1.2 & 1.4 \\
\hline & \multirow{3}{*}{$\hat{y}_{\sigma 1}$} & \multirow{3}{*}{ STB } & 1 & - & 0.2 & 0.5 \\
\hline & & & 2 & - & 0.1 & 0.5 \\
\hline & & & 3 & - & 0.1 & 0.2 \\
\hline \multirow{6}{*}{ Width } & \multirow{3}{*}{$\hat{y}_{\mu 2}$} & \multirow{3}{*}{ NTB } & 1 & 1.7 & 6.6 & 7.9 \\
\hline & & & 2 & 6.5 & 7.3 & 8.2 \\
\hline & & & 3 & 4.0 & 4.5 & 5.0 \\
\hline & \multirow{3}{*}{$\hat{y}_{\sigma 2}$} & \multirow{3}{*}{ STB } & 1 & - & 1.0 & 3.1 \\
\hline & & & 2 & - & 0.4 & 0.9 \\
\hline & & & 3 & - & 0.2 & 0.5 \\
\hline
\end{tabular}




\begin{tabular}{|c|c|c|c|c|c|c|}
\hline \multirow{6}{*}{ Left Toe Angle } & \multirow{3}{*}{$\hat{y}_{\mu 3}$} & \multirow{3}{*}{ NTB } & 1 & 144.9 & 159.4 & 170.1 \\
\hline & & & 2 & 139.1 & 148.2 & 157.4 \\
\hline & & & 3 & 124.5 & 132.7 & 141.3 \\
\hline & \multirow{3}{*}{$\hat{y}_{\sigma 3}$} & \multirow{3}{*}{ STB } & 1 & - & 3.3 & 12.6 \\
\hline & & & 2 & - & 2.7 & 9.1 \\
\hline & & & 3 & - & 3.2 & 8.4 \\
\hline \multirow{6}{*}{$\begin{array}{l}\text { Right Toe } \\
\text { Angle }\end{array}$} & \multirow{3}{*}{$\hat{y}_{\mu 4}$} & \multirow{3}{*}{ NTB } & 1 & 129.5 & 158.9 & 165.0 \\
\hline & & & 2 & 137.5 & 148.6 & 152.9 \\
\hline & & & 3 & 126.6 & 135.7 & 146.6 \\
\hline & \multirow{3}{*}{$\hat{y}_{\sigma 4}$} & \multirow{3}{*}{ STB } & 1 & - & 3.7 & 17.8 \\
\hline & & & 2 & - & 2.8 & 8.7 \\
\hline & & & 3 & - & 3.3 & 10.0 \\
\hline
\end{tabular}

Based on the parameter values given in Table 5, we construct eight individual desirability functions, as shown in Figure 9. For simplicity, we assume linear functions for the desirability functions. Three sets of the eight functions can be defined because we have three types of beads, as shown in Table 5 .
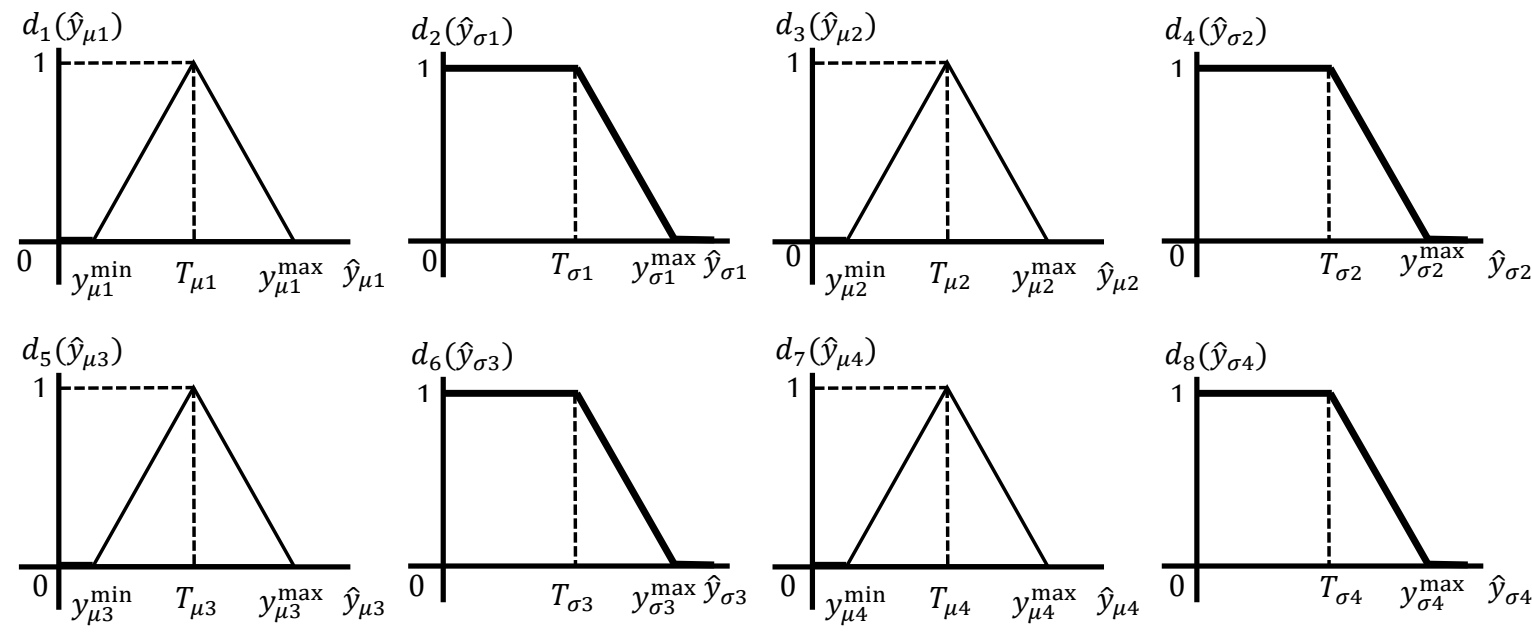

Fig. 9 The eight individual desirability functions

\subsubsection{Step 6: Maximizing the desirability function}

The eight individual desirability functions given in Figure 9 are aggregated into an overall desirability function, and then the optimal setting of the input variable is obtained by maximizing the overall desirability function. We assume that the eight desirability functions are equally important; thus, we simply aggregate them as a geometric mean as follows: 


$$
D(\mathbf{x})=\left(\prod_{j=1}^{8} d_{j}(\mathbf{x})\right)^{\frac{1}{8}}
$$

Table 6 reports the result of maximizing the overall desirability function. It is carried out using the Minitab 19 software. The maximization is carried out for each bead; thus, three sets of optimal settings of the input variables are obtained: $D$ is highest when Bead 1 is used as the target bead geometry, and $D$ is close to 1 , which means that it is capable to obtain Bead 1 by setting the input variable values into the optimal setting. Conversely, it is relatively difficult to obtain Bead 3 according to the optimization results.

Table 6 Optimization results

\begin{tabular}{|c|c|c|c|c|c|c|c|c|c|c|c|c|c|c|c|}
\hline \multirow{3}{*}{ Bead } & \multicolumn{6}{|c|}{ Optimal setting } & \multirow{2}{*}{\multicolumn{8}{|c|}{ Predicted response value at the optimal setting }} & \multirow{3}{*}{$D$} \\
\hline & \multicolumn{3}{|c|}{ Coded } & \multicolumn{3}{|c|}{ Real } & & & & & & & & & \\
\hline & $x_{1}$ & $x_{2}$ & $x_{3}$ & $x_{1}$ & $x_{2}$ & $x_{3}$ & $\hat{y}_{\mu 1}$ & $\hat{y}_{\sigma 1}$ & $\hat{y}_{\mu 2}$ & $\hat{y}_{\sigma 2}$ & $\hat{y}_{\mu 3}$ & $\hat{y}_{\sigma 3}$ & $\hat{y}_{\mu 4}$ & $\hat{y}_{\sigma 4}$ & \\
\hline 1 & 1.01 & 0.84 & -0.09 & 323 & 18.80 & 146 & 1.10 & 0.11 & 6.17 & 0.49 & 158.08 & 2.93 & 158.75 & 2.69 & 0.98 \\
\hline 2 & 0.64 & -0.20 & 0.75 & 315 & 14.11 & 185 & 1.66 & 0.13 & 6.80 & 0.34 & 148.30 & 2.81 & 148.57 & 2.79 & 0.82 \\
\hline 3 & -1.27 & -1.23 & -0.83 & 271 & 9.42 & 112 & 1.18 & 0.13 & 4.40 & 0.35 & 136.38 & 5.22 & 135.60 & 3.83 & 0.74 \\
\hline
\end{tabular}

\subsubsection{Step 7: Validating the result of the optimization}

In Step 7, the optimal settings obtained in Step 6 are validated. Usually, additional experiments are conducted by maintaining the input variables at the optimal settings. Then, the measured response values from the additional experiments are compared to the predicted response values. If they are similar, it is believed that the obtained optimal setting is valid. However, we could not conduct additional experiments by keeping the input variables at the optimal settings because they were difficult to precisely control. Therefore, we decided to validate the optimal settings indirectly by validating the eight response surface models.

It is important to validate the eight response surface models because the optimal setting of the input variables is obtained based on the eight response surface models, assuming that these models are fitted reasonably well. More specifically, the eight response surface models are transformed into eight individual desirability functions, which are aggregated into the overall desirability function. The optimal setting is obtained by maximizing the overall desirability 
function. Thus, when the assumption is not satisfied, the obtained optimal setting is far from the true optimal setting [16].

To validate the eight response surface models, we conduct six additional experiments, and six beads are obtained thereof. Thereafter, we measure the height, width, and left and right angles at 100 points on each bead, as in Step 2. The rightmost eight columns in Table 7 present the mean and standard deviations of the measured values for the four geometries. For validation, we also calculate the predicted mean and standard deviation of the bead geometry from the eight response surface models.

Table 7 Experimental conditions and results

\begin{tabular}{|c|c|c|c|c|c|c|c|c|c|c|c|c|c|c|c|c|c|c|c|}
\hline \multirow{2}{*}{$\begin{array}{l}\text { Experiment } \\
\text { number }\end{array}$} & \multicolumn{3}{|c|}{$\begin{array}{l}\text { Experimental } \\
\text { conditions }\end{array}$} & \multicolumn{8}{|c|}{$\begin{array}{l}\text { "Predicted" mean and standard deviation } \\
\text { of bead geometry }\end{array}$} & \multicolumn{8}{|c|}{$\begin{array}{c}\text { "Actual" mean and standard deviation } \\
\text { of bead geometry }\end{array}$} \\
\hline & $x_{1}$ & $x_{2}$ & $x_{3}$ & $\hat{y}_{\mu 1}$ & $\hat{y}_{\sigma 1}$ & $\hat{y}_{\mu 2}$ & $\hat{y}_{\sigma 2}$ & $\hat{y}_{\mu 3}$ & $\hat{y}_{\sigma 3}$ & $\hat{y}_{\mu 4}$ & $\hat{y}_{\sigma 4}$ & $y_{\mu 1}$ & $y_{\sigma 1}$ & $y_{\mu 2}$ & $y_{\sigma 2}$ & $y_{\mu 3}$ & $y_{\sigma 3}$ & $y_{\mu 4}$ & $y_{\sigma 4}$ \\
\hline 1 & 275 & 15 & 150 & 50 & 0.18 & 5.11 & 0.31 & 142 & 4.60 & 140 & 3.23 & 1.76 & 0.20 & 5.85 & 0.80 & 124 & 3.74 & 129 & 3.50 \\
\hline 2 & 325 & 15 & 150 & 1.21 & 0.22 & 6.64 & 0.46 & 156 & 3.05 & 156 & 2.97 & 1.50 & 0.26 & 7.16 & 0.57 & 135 & 5.40 & 139 & 4.15 \\
\hline 3 & 300 & 10 & 150 & 1.43 & 0.23 & 6 & 0.43 & 14 & 4.29 & 144 & 3 & 2.05 & 0.30 & 7.81 & 0.59 & 114 & 4.02 & 126 & 4.43 \\
\hline 4 & 300 & 20 & 150 & 1.20 & 0.22 & 5.69 & 0.45 & 155 & 3.40 & 155 & 3.24 & 1.40 & 0.25 & 5.90 & 0.58 & 137 & 4.86 & 141 & 3.10 \\
\hline 5 & 300 & 15 & 100 & 0.93 & 0.20 & 5.82 & 0.09 & 162 & 3.20 & 161 & 2.90 & 1.05 & 0.22 & 6.49 & 0.54 & 145 & 3.27 & 148 & 3.55 \\
\hline 6 & 300 & 15 & 200 & 1.89 & 0.14 & 6.35 & 0.34 & 142 & 3.39 & 142 & 2.81 & 2.08 & 0.21 & 8.04 & 1.13 & 132 & 4.55 & 133 & 2.56 \\
\hline
\end{tabular}

Based on the predicted and actual values, we calculate the mean absolute percentage error (MAPE) to evaluate the performance of the response surface models. This is an indicator of the amount of error that constitutes the predicted value. The MAPE has values ranging from 0 to 100 , and the closer it is to zero, the better the performance of the response surface model. When $n$ samples are present, $A_{t}$ is the actual value and $F_{t}$ is the predicted value. In our example, we have six samples (i.e., $n=6$ ) because we conduct six additional experiments. Table 8 lists the MAPE values of the eight response surface models. The eight models show generally low MAPE values, which means that the response surface models are reasonably well-fitted.

$$
M A P E=\frac{100}{n} \sum_{t=1}^{n}\left|\frac{A_{t}-F_{t}}{A_{t}}\right|
$$


Table 8 MAPE of the eight response surface models

\begin{tabular}{ccccccccc}
\hline & $\hat{y}_{\mu 1}$ & $\hat{y}_{\sigma 1}$ & $\hat{y}_{\mu 2}$ & $\hat{y}_{\sigma 2}$ & $\hat{y}_{\mu 3}$ & $\hat{y}_{\sigma 3}$ & $\hat{y}_{\mu 4}$ & $\hat{y}_{\sigma 4}$ \\
\hline MAPE & 16.4 & 17.2 & 12.4 & 27.9 & 14.9 & 21.8 & 10.2 & 11.9 \\
\hline
\end{tabular}

\section{Concluding remarks}

In this study, we investigate to determine the optimal process parameters for the ideal beads defined by users in welding using TZM materials. We systematically consider the tradeoffs between the eight responses vis-à-vis the bead geometry by employing the desirability function method. Furthermore, TZM single-layer welding is performed under the designed settings (i.e., CCD), the bead geometry is measured, the response surface model is established, and the individual and overall desirability functions are established to optimize the optimal process parameters for each ideal bead. This study establishes optimal parameters that minimize variability in beads, considering not only the mean of the bead geometry but also the standard deviation, in the process of determining optimal process parameters. The plausibility of the study is proven by validating the eight response surface models.

\section{Declarations}

a. Funding: This research was supported by the MSIT (Ministry of Science, ICT), Korea, under the High-Potential Individuals Global Training Program) (No. 2020-0-01539) supervised by the IITP (Institute for Information \& Communications Technology Planning \& Evaluation).

b. Conflicts of interest/Competing interests: The authors declare no competing interests.

c. Availability of data and material: Not applicable.

d. Code availability: Not applicable.

e. Ethics approval: The submitted work is original, complete and has not been submitted/published elsewhere in any form or language.

f. Consent to participate: Not applicable.

g. Consent for publication: The authors provide their consent to publish the manuscript in the International Journal of Advanced Manufacturing Technology.

\section{References}


[1] Kim D, Rhee S, Park H (2002) Modelling and optimization of a GMA welding process by genetic algorithm and response surface methodology. International Journal of Production Research. 40, 1699-1711. https://doi.org/10.1080/00207540110119964

[2] Dey V, Pratihar DK, Datta GL, Jha MN, Saha TK, Bapat AV (2009) Optimization of bead geometry in electron beam welding using a Genetic Algorithm. Journal of $\begin{array}{llll}\text { Materials } & \text { Processing } & \text { Technology. } & \text { 209, }\end{array}$ https://doi.org/10.1016/j.jmatprotec.2008.03.019

[3] Geng H, Li J, Xiong J, Lin X, Zhang F (2017) Optimization of wire feed for WAAM based additive manufacturing. Journal of Materials Processing Technology. 243, 4047. https://doi.org/10.1016/j.jmatprotec.2016.11.027

[4] Benyounis KY, Olabi AG, Hashmi MSJ (2005) Effect of laser welding parameters on the heat input and weld-bead profile. Journal of Materials Processing Technology. 164, 978-985. https://doi.org/10.1016/j.jmatprotec.2005.02.060

[5] Gunaraj V, Muruga N (1999) Application of response surface methodology for predicting weld bead quality in submerged arc welding of pipes. Journal of Materials Processing Technology. 88, 266-275. https://doi.org/10.1016/S0924-0136(98)004051

[6] Lee DH, Kim SH, Byun JH (2020) A method of steepest ascent for multiresponse surface optimization using a desirability function method. Quality and Reliability Engineering International. 36, 1931-1948 https://doi.org/10.1002/qre.2666

[7] Lee DH, Jeong IJ, Kim KJ (2018) A desirability function method for optimizing mean and variability of multiple responses using a posterior preference articulation approach. Quality and Reliability Engineering International, 34, 360-376. https://doi.org/10.1002/qre.2258

[8] Derringer G, Suich R (1980) Simultaneous optimization of several response variables. Journal of Quality Technology. 12, 214-219. https://doi.org/10.1080/00224065.1980.11980968

[9] Box GE, Wilson KB (1951) On the experimental attainment of optimum settings. J. of the Roy. Statistical Soc.: Ser. B (Methodological). 13, 1-45.

[10] Myers RH, Montgomery DC, Anderson-Cook CM (2016) Response surface methodology: Process and product optimization using designed experiments. John 
Wiley \& Sons.

[11] Saha P, Waghmare D (2020) Parametric optimization for autogenous butt laser welding of sub-millimeter thick SS 316 sheets using central composite design. Optics \& Lase Technology. 122, 105833. https://doi.org/10.1016/j.optlastec.2019.105833

[12] Panaskar N, Terkar RP (2020) Optimization of friction stir welding process parameters for AA6063-ETP copper using central composite design. World Journal of Engineering. 17, 491-507. https://doi.org/10.1108/WJE-11-2019-0322

[13] Pignatiello JJ (1993) Strategies for robust multiresponse quality engineering. IIE Transactions. 25, 5-15. https://doi.org/10.1080/07408179308964286

[14]Vining GG (1998) A compromise Approach to Multiresponse Optimization. Journal of Quality Technology. 30 309-313. https://doi.org/10.1080/00224065.1998.11979867

[15] Kim KJ, Lin D (1998) Dual Response Surface Optimization: A Fuzzy Modeling Approach. Journal of Quality Technology. 30, 1-10. https://doi.org/10.1080/00224065.1998.11979814

[16] Xu D, Albin SL (2003) Robust Optimization of Experimentally Derived Objective Functions. IIE Transactions. 35, 793-802. https://doi.org/10.1080/07408170304408 